\title{
Similarity between concurrent visual discriminations: Dimensions and objects
}

\author{
JOHN DUNCAN \\ MRC Applied Psychology Unit, Cambridge, England
}

\begin{abstract}
Accuracy is often reduced when two visual discriminations must be made concurrently ("divided attention"). According to a hypothesis originally proposed by Treisman (1969) and Allport (1971), this result should depend on the similarity of required discriminations. When discriminations concern different visual dimensions, they should be made in somewhat separate visual subsystems, reducing interference between them. This prediction was tested in two experiments, involving discriminations of shape, size, orientation, and spatial frequency. In different conditions of divided attention, concurrent discriminations concerned either the same or different dimensions, and either one or two objects. The results showed that performance depends only on the number of relevant objects, not on the number or similarity of required discriminations. They suggest that selective attention to an object is a coordinated state in which the outputs of multiple visual subsystems are made concurrently available for control of behavior.
\end{abstract}

In vision, there is often a cost to "dividing attention"; performance suffers when two visual discriminations must be made at once (e.g., Broadbent, 1971; Treisman, 1969). This paper concerns the sensitivity of such performance decrements to two different kinds of similarity between the discriminations that must be made.

First, concurrent discriminations might concern the same or different objects. For example, a subject might be asked to identify both the shape and color of one object, or the shape of one and the color of another (Lappin, 1967; Treisman, 1969). This is a potent variable influencing the size of performance decrement. In some cases, it has been shown that two discriminations concerning the same briefly presented object can be made simultaneously without loss of accuracy, whereas discriminations concerning two different objects cannot ${ }^{1}$ (Duncan, 1984, in press; see also Kahneman \& Henik, 1977; Treisman, Kahneman, \& Burkell, 1983). Such findings suggest a model in which early Gestalt grouping operations segment the visual input into packages corresponding to discrete objects. Interference occurs in divided attention because some subsequent, selective processing system is sensitive to the number of objects it must deal with (Broadbent, 1958; Neisser, 1967); single objects, however, can be selected as wholes.

The main focus of this paper is a second form of similarity: Concurrent discriminations might concern the same or different dimensions. More than 20 years ago, Treisman (1969) and Allport (1971) suggested that dimensions such as shape and color might be processed by separate perceptual "analyzers," and that interference between

This research was supported by a grant from the Human Frontier Science Program. Address correspondence to J. Duncan, MRC Applied Psychology Unit, 15 Chaucer Road, Cambridge CB2 2EF, England. concurrent discriminations might arise only when they involved the same analyzer. Two color or two shape discriminations would show substantial interference, but one color and one shape discrimination would not. In fact, there are many good reasons to suppose that the different dimensions of a visual stimulus are processed by at least somewhat separate mechanisms. Physiologically, information concerning various different dimensions (e.g., motion and color) is segregated into a number of separate processing streams, beginning as early as the retina and continuing into separate subdivisions of striate and extrastriate cortex (e.g., Desimone \& Ungerleider, 1989; Livingstone \& Hubel, 1987). Computationally, quite different kinds of operation are needed in the analysis of different visual dimensions (e.g., Ullman, 1984). Behaviorally, the constancies and related phenomena indicate the importance of discarding one type of information (e.g., size or location) in the analysis of another (e.g., shape).

Of course, the simple existence of separate visual subsystems - devoted at least in part to the analysis of different visual dimensions-does not in itself guarantee the sort of functional independence that is suggested by the Treisman-Allport hypothesis. The hypothesis implies that subsystems can work in parallel without mutual interference, and also that they can be separately controlled such that the shape analyzer, for example, can work on one object while the color analyzer works on another. Such independent control is denied by the object model. According to that model, an object's different attributes may be processed by separate subsystems, but selective attention is then a coordinated state in which the outputs of multiple subsystems, describing these various separate attributes, are made available together for the control of behavior (e.g., Treisman \& Souther, 1985). For that reason, there will always be interference when concurrent 
discriminations concern different objects, whether or not they concern the same visual dimension.

Though two early experiments (Allport, 1971; Wing \& Allport, 1972) seemed to support the analyzer hypothesis, their displays made it difficult to separate similarity of dimensions from the involvement of one or two objects. For example, Wing and Allport found interference between two orientation discriminations, but no interference between discriminations of orientation and spatial frequency. According to one plausible interpretation of their display (a patch of grating with a bar lying across it), however, the two orientation discriminations concerned different objects, while the orientation and frequency judgments concerned the same object (for a fuller discussion, see Duncan, 1984). Subsequently, there have been several experiments showing some interference even when discriminations concern very different visual dimensions (e.g., Duncan, 1984), but few direct comparisons of same- and different-dimension cases.

Recently, Duncan (in press) reported a study of one case-concurrent discriminations of spatial location and various different object features-for which the idea of distinct perceptual analyzers is especially well motivated. Physiologically, there are strong reasons to distinguish an occipitoparietal system involved especially in the analysis of spatial relations, and an occipitotemporal system involved especially in the analysis of object features leading to object recognition (Ungerleider \& Mishkin, 1982). Of course, successful object recognition requires location constancy, or discarding of information concerning where an object happens to be located. Duncan's results, however, provided no support for the analyzer hypothesis. Two discriminations (one of location, one of an object feature such as size, orientation, or spatial frequency) concerning the same briefly presented object could be made concurrently without mutual interference, but discriminations concerning two different objects could not. In fact, interference in the two-object case was precisely the same whether concurrent discriminations concerned the same or different dimensions (two spatial or two feature discriminations vs. one of each).

On several grounds, however, one might suspect that location is an unusual dimension in vision. Location has often been seen as the "medium"' of visual perception (Kubovy, 1981), in that, for example, shared location may be the vehicle by which an object's multiple attributes are combined (Nissen, 1985). The present experiments, accordingly, attempt to generalize Duncan's (in press) results by dealing with a range of other dimensions. The dimensions examined here-shape, size, frequency, and orientation-concern visual contours; a companion paper will deal with surface characteristics such as color and brightness.

\section{EXPERIMENT 1}

Experiment 1 concerned the size and shape of letters. Independent size and shape analyzers are plausible because, for example, shape recognition requires size con- stancy. Correspondingly, many cells in the inferotemporal cortex - the culmination of the shape-recognition systemrespond to the same preferred shapes, irrespective of size (e.g., Schwartz, Desimone, Albright, \& Gross, 1983).

Two letters, varying independently in shape and size, were presented side by side. In different conditions of divided attention, subjects identified (1) the size and shape of a single letter, (2) the same attribute (size or shape) of both letters, (3) the shape of one and size of the other, or (4) both attributes of both objects. The hypothesis that performance depends only on the number of relevant objects ${ }^{2}$ predicts the highest accuracy in Condition 1, and equal decrements in Conditions $2-4$. The analyzer hypothesis, in contrast, predicts better performance in Condition 3 than in 2 or 4.

The experiment is also relevant to a different theoretical suggestion. It has sometimes been proposed that, at least in part, selective attention in vision is a matter of selecting a certain range of spatial frequencies for visual analysis (Shulman \& Wilson, 1987; Watt, 1988). This view makes a prediction that contrasts with the analyzer hypothesis: Decrements in divided attention will be stronger when the spatial frequencies underlying concurrent discriminations are more different. Though it would be difficult to specify exactly which spatial frequencies contributed to shape and size judgments, the two size judgments in particular were identical and so, according to this hypothesis, should have been very easy to perform together, whereas concurrent size and shape judgments would have involved somewhat different frequencies and so should have been more difficult.

\section{Method}

Subjects. Twenty-eight paid subjects, aged between 18 and 39 years, were recruited from the Applied Psychology Unit panel. Twenty-three were female.

Task. Displays were presented on an X-Y oscilloscope (P31 phosphor) controlled by a Cambridge Electronic Design laboratory computer. The room was semidarkened. A chinrest was used to fix the viewing distance at about $45 \mathrm{~cm}$.

Each trial began with a fixation point in the center of the screen. Central fixation was emphasized in the instructions, but was not measured. When ready, the subject pressed a key to give an immediate $60-\mathrm{msec}$ presentation of the stimulus display. This display consisted of two letters positioned symmetrically, one to the left and one to the right of the fixation point. The letters were centered on the horizontal meridian, and were separated center-to-center by either $0.5^{\circ}$ (14 subjects) or $5^{\circ}$ (14 subjects). The display was immediately followed by a 500-msec mask (a pair of " @”" characters presented at the same locations as the preceding letters), after which time the fixation point returned for the next trial.

The letters were drawn in a standard $5 \times 7$ pixel font. The left letter was either a $C$ or a $G$, and measured either $36^{\prime} \times 21^{\prime}$ or $26^{\prime} \times 16^{\prime}$ of visual arc. The right letter was either an $E$ or an $F$, with the same two alternative sizes. The mask character was drawn at the larger of the two sizes.

Two-alternative forced-choice responses for each relevant dimension were made vocally. The answers were written down by the experimenter and later typed into the computer for scoring. The subjects were told to proceed at their own speed, maximizing accuracy.

Design. Each subject served in seven conditions, organized conceptually into four groups. In two conditions (termed 1[2]), the sub- 
jects identified the two attributes of a single object-left in one condition, right in the other. In two other conditions $(2[2 s])$, the subjects identified the same attribute of both objects-size in one condition, shape in the other. In two others $(2[2 \mathrm{~d}])$, the subjects identified different attributes of the two objects-size of the left and shape of the right in one condition, vice versa in the other. In the final condition (2[4]), the subjects identified both attributes of both objects. In all cases, responses were required in a fixed order, left object before right, and within an object, size before shape.

On each of 2 days, the subjects carried out one block of trials per condition, with the order of conditions counterbalanced within each group of 14 subjects. The blocks were divided into two subblocks separated by a 10 -sec pause; the first had 24 trials and the second had 48 trials. Data were analyzed only from 48 -trial subblocks of the second session. Within these blocks, each of the four possible stimuli on each side appeared equally often, in an otherwise random order.

\section{Results}

The mean proportions correct for size and shape judgments in each condition are shown in Table 1 . Values are means across left and right letters, and across spatial separation.

The data were examined by analysis of variance (ANOVA), with separation $\left(0.5^{\circ}, 5^{\circ}\right)$ as a betweensubjects factor, and condition (1[2], 2[2s], 2[2d], 2[4]), side (left, right), and dimension (size, shape) as withinsubjects factors. Of most interest was the significant main effect of condition $[F(3,78)=10.4, p<.001]$. By Newman-Keuls, Condition 1[2] differed from each of the other three $(p<.01$, in each case), but no other difference was significant. These results support the hypothesis that the difficulty of divided attention depends only on the number of relevant objects, not on the number or (dimensional) similarity of required discriminations.

There was a significant main effect of dimension $[F(1,26)$ $=17.1, p<.001]$, with size judgments being less accurate than shape. There was also a significant main effect of separation $[F(1,26)=4.3, p<.05]$. Overall accuracy was .077 higher with $0.5^{\circ}$ separation; that is, for objects closer to the fixation point. Though the difference between Condition 1[2] and the other conditions was somewhat greater with $5^{\circ}(.070)$ than with $0.5^{\circ}(.039)$ separation, the interaction between separation and condition was far from significant $[F(3,78)=0.8]$. Instead, there was a significant three-way interaction between separation, side, and condition $[F(3,78)=4.9, p<.01]$, modulating a significant two-way interaction between the latter two $[F(3,78)=4.7, p<.01]$. With $0.5^{\circ}$ separation, the difference between Condition 1[2] and the other conditions was almost exactly the same for left and right objects, but with $5^{\circ}$ separation, this difference was con-

Table 1

Mean Proportion Correct for Experiment 1

\begin{tabular}{lcccc}
\hline & \multicolumn{4}{c}{ Condition } \\
\cline { 2 - 5 } & $1[2]$ & $2[2 \mathrm{~s}]$ & $2[2 \mathrm{~d}]$ & $2[4]$ \\
\hline Size & .715 & .651 & .626 & .672 \\
Shape & .784 & .744 & .733 & .745 \\
$M$ & .749 & .697 & .679 & .708 \\
\hline
\end{tabular}

centrated entirely in the right (second-reported) object. Very similar results were reported by Eriksen and Colegate (1971), suggesting that decrements due to divided attention are sometimes spread between close objects, but focused on the second-reported of two distant objects.

\section{Discussion}

The most important result in this experiment was equal performance in the three conditions requiring divided attention between two objects. There was no evidence for independent processing in size and shape systems, as would have been reflected in better performance for Condition $2[2 \mathrm{~d}]$ than for $2[2 \mathrm{~s}]$ or $2[4]$. There was no reliable evidence for better performance when all the discriminations involved the same spatial frequencies (Condition $2[2 \mathrm{~s}]$ ). There was no evidence for memory losses or other limitations dependent on the number of judgments reported (Condition 2[4] vs. the other two), at least up to four. The results were just the same, furthermore, whether the two discriminations to be made on one dimension were identical (size judgments on left and right) or different (shape).

A possible concern is that equal performance in Conditions $2[2 \mathrm{~s}]$ and 2[2d] may have reflected chance offsetting between two opposed factors, partial independence between size and shape analyzers, but also a preference for two discriminations involving the same spatial frequencies. This view is unlikely, however, because performance in Condition 2[4] should in that case have been worse, involving joint use of both analyzers on both objects, and also maximal spread of relevant spatial frequencies.

Of lesser importance is the difference obtained between Condition 1[2] and the other conditions. In agreement with previous results, this finding suggests that divided attention becomes more difficult as the number of relevant objects increases; in this experiment, however, there was no objective control of fixation, making it possible that the subjects shifted their gaze toward the single relevant object in Condition 1[2]. This issue is addressed again in Experiment 2.

\section{EXPERIMENT 2}

The major results of Experiment 1 rested on a failure to reject the null hypothesis, so a replication with new stimuli and different elementary visual dimensions was attempted in Experiment 2. The new stimuli were patches of grating, varying in orientation, length, and spatial frequency.

\section{Method}

Subjects. Thirty-six paid subjects, aged between 18 and 45 years, were recruited as in Experiment 1. Twenty-eight were female.

Task. In this experiment, the displays were presented on a Hewlett-Packard X-Y display (1332A) with P24 phosphor. Viewing distance, fixed as before, was about $65 \mathrm{~cm}$. A typical display is shown in Figure 1. One patch of lines (the horizontal patch) was centered $1^{\circ} 29^{\prime}$ of visual arc unpredictably either to left or right of the fixation point; another (the vertical patch) was centered $1^{\circ} 29^{\prime}$ 


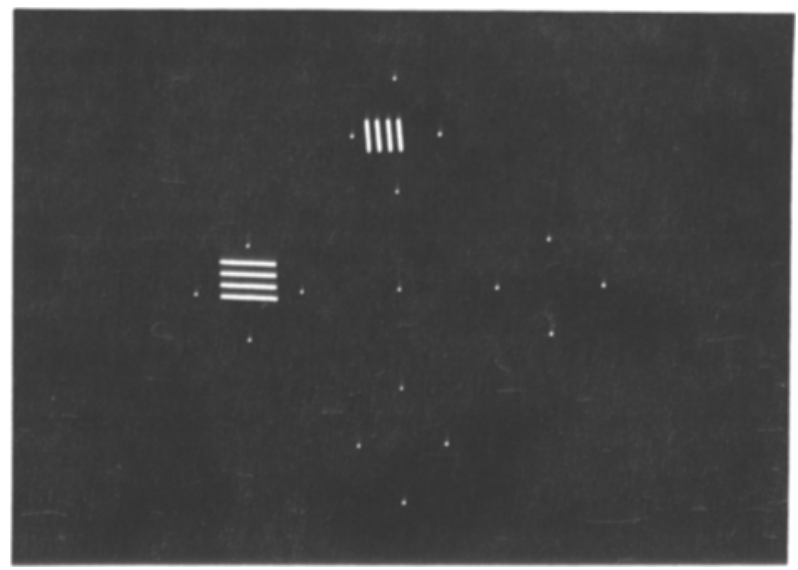

Figure 1. Example display for Experiment 2.

above or below fixation. Unpredictable positions were used simply to ensure central fixation at display onset; position was always irrelevant to the subject's task. Possible positions were indicated by $1^{\circ} 4^{\prime}$ " "boxes" of four dots (see Figure 1), present throughout the trial.

For any given subject, each patch varied across trials in one and only one attribute (the relevant attribute). Divided attention or dualdiscrimination blocks (relevant attributes of both horizontal and vertical patches to be identified on each trial) were compared with focused attention or single-discrimination controls (relevant attribute of only the horizontal or only the vertical patch identified for a block of trials). Experiment 2A (12 subjects) dealt with orientation and length. Relevant attributes for horizontal and vertical patches, respectively, were: orientation and orientation ( 3 subjects), length and length ( 3 subjects), orientation and length ( 3 subjects), and length and orientation ( 3 subjects). In Experiments $2 B$ and $2 C$, this design was repeated, using orientation and spatial frequency in Experiment $2 \mathrm{~B}$, and length and spatial frequency in Experiment $2 \mathrm{C}$. Altogether, then, there were 18 subjects whose two relevant dimensions were the same, and 18 whose two relevant dimensions were different.

When varying, orientation took values of $3^{\circ} 48^{\prime}$ clockwise or counterclockwise from either horizontal (horizontal patch) or vertical (vertical patch). Length took values of $31^{\prime}$ or $47^{\prime}$ of arc (horizontal patch), and $17^{\prime}$ or $25^{\prime}$ of arc (vertical patch). Spatial frequency for both patches took values of 9 or 12 cycles/degree (cpd) (total patch width $=20^{\prime}$ of arc, four or five lines per patch). When fixed, horizontal-patch orientation was clockwise and vertical-patch orientation was counterclockwise, length was the shorter value for each patch, and spatial frequency was $12 \mathrm{cpd}$. Note that, for a given subject, both relevant attributes (one per patch) always varied, even in single-discrimination blocks. For comparability with Duncan's (1992) stimuli, the horizontal patch was always centered $8^{\prime}$ of arc above the midline within its box, and the vertical was centered $8^{\prime}$ of arc to the left of midline.

Each trial began with the central fixation point and four boxes indicating possible stimulus positions. When the subject pressed a foot switch, the display of two line patches was immediately presented for a predetermined exposure duration, followed at once by a 500-msec masking character in each of the two locations containing a stimulus on that trial. Masking characters were dense patches of jumbled contour, each measuring $58^{\prime} \times 44^{\prime}$ of arc. Following the mask, the display went blank until the response was eomplete.
Responses for the horizontal patch were made with a pair of keys operated by the middle and forefingers of the left hand. Within this pair of keys, response assignments for the different dimensions were: orientation-left key for top of patch pointing left, right key for top of patch pointing right; length-left key for small, right key for large; and spatial frequency-left key for high, right key for low. Responses for the vertical patch were made on an equivalent pair of keys operated by the right hand, with the same response assignments. When both responses were required (dualdiscrimination blocks), the left-hand response (horizontal patch) was required first. Care was taken to ensure that mappings were thoroughly learned before the experiment began, and the subjects were told to take as much time as they needed to ensure that response errors never occurred. ${ }^{3}$ After a response was complete, there was a blank interval of $1 \mathrm{sec}$ before onset of the fixation display for the next trial.

Design. Each subject served in a single session of about $1 \mathrm{~h}$. In an initial practice phase, there was one block of trials per condition, with the order of the three conditions (dual-discrimination, single horizontal, single vertical) counterbalanced within each group of 3 subjects. Each block was divided into subblocks of 12 trials with a stimulus exposure of $250 \mathrm{msec}, 12$ with an exposure of $150 \mathrm{msec}$, and 24 with an exposure of $100 \mathrm{msec}$, in that order. Following this practice phase, a single, fixed exposure duration between 100 and 150 msec was chosen for the remainder of the session, in an attempt to keep proportion correct above .800 in singlediscrimination blocks. Mean exposure was $127 \mathrm{msec}$ for the subjects whose two relevant dimensions were the same, and $129 \mathrm{msec}$ for those whose two relevant dimensions were different. Data were then collected in an additional one block of trials per condition, with the same order of conditions as before. Each block was divided into two subblocks, the first with 56 trials (of which the first 8 were discarded) and the second with 48. Across the 96 scored trials per condition, each combination of relevant-dimension values and patch positions occurred equally often, in an otherwise random order. At the end of each practice or experimental subblock, percent correct for that subblock was shown on the screen for $10 \mathrm{sec}$.

\section{Results}

The mean results are shown in Table 2 . These are averages across Experiments $2 \mathrm{~A}-2 \mathrm{C}$; thus, orientation data are derived from Experiments $2 \mathrm{~A}$ and $2 \mathrm{~B}$, length from $2 B$ and $2 C$, and frequency from $2 B$ and $2 C$. All data were combined into a single ANOVA, with the between-subjects factor of similarity (same vs. different relevant dimensions) and the within-subjects factors of patch (horizontal vs. vertical) and number of discriminations (single vs.

Table 2

Mean Proportion Correct for Experiment 2

\begin{tabular}{lccccc}
\hline & \multicolumn{2}{c}{ Horizontal } & & \multicolumn{2}{c}{ Vertical } \\
\cline { 2 - 3 } \cline { 5 - 5 } & Single & Dual & & Single & Dual \\
\hline \multirow{4}{*}{ Orientation } & .927 & .880 & .830 & .535 \\
Length & .941 & .797 & .801 & .656 \\
Frequency & .875 & .837 & .797 & .646 \\
$M$ & .914 & .838 & .809 & .612 \\
& \multicolumn{3}{c}{ Same Dimension } \\
Orientation & .978 & .903 & .783 & .580 \\
Length & .948 & .924 & .787 & .649 \\
Frequency & .837 & .776 & .854 & .717 \\
$M$ & .921 & .868 & .808 & .649 \\
\hline
\end{tabular}


dual). Of major interest was the main effect of number of discriminations $[F(1,34)=114.7, p<.001]$, which showed no interaction with similarity $[F(1,34)=1.8]$. Dividing attention between discriminations, and hence objects, produced a mean loss of accuracy of .121 , which was roughly the same whether the two discriminations concerned the same (.137) or different (.106) dimensions. The small difference between these two is opposite that obtained in Experiment 1 (Conditions 2[2s] vs. 2[2d]). Again, these data suggest that accuracy was limited entirely by the number of relevant objects, irrespective of whether discriminations involved the same or different dimensions.

Final significant effects were a main effect of patch $[F(1,34)=157.6, p<.001]$ and its interaction with number of discriminations $[F(1,34)=34.2, p<.001]$. Discriminations were more accurate for the horizontal than for the vertical patch, and were also less influenced by divided attention. Previous data have also shown that divided attention decrements are often stronger on whichever discrimination is reported second (Duncan, 1984, 1992).

Three similar ANOVAs were conducted on the separate data from Experiments $2 \mathrm{~A}-\mathrm{C}$. In no case was the interaction between similarity and number of discriminations significant, though for Experiment $2 \mathrm{~A}$ it was close $[F(1,10)=4.6, p<.06]$; the results in this case tending in the direction predicted by the analyzer hypothesis. In Experiment 2C, discriminations of length and frequency should have differed markedly in the spatial frequencies they depended upon; here, however, the loss of accuracy in the dual-discrimination conditions was almost exactly the same whether required discriminations were the same $(.131)$ or different $(.127)$.

\section{Discussion}

In this experiment, there was a large effect of number of discriminations, confirming-this time with no possible confound from eye movements-the large cost that is consequent upon dividing attention between objects (Duncan, 1984 , in press). In contrast, any effect of similarity in dimension was small and nonsignificant, as well as being opposite to the nonsignificant trend in Experiment 1.

Again, there was no support for the idea that concurrent discriminations are most difficult when they concern different spatial frequencies. In general, the idea of selective attention to a particular range of frequencies seems only marginally relevant to the main phenomena of visual selection. For example, in an experiment by Rock and Gutman (1981), overlapping red and green shapes were presented at fixation. Subjects attending selectively to the shape of one color revealed little knowledge of the other, even though the two were much the same in construction and overall dimensions. Similarly, in partial report experiments, subjects select letters from briefly presented arrays on the basis of such characteristics as location or color, again with little awareness of letters that are re- jected (von Wright, 1968). In such cases, selection is clearly a matter of choosing objects, not spatial frequencies.

\section{GENERAL DISCUSSION}

In general, these results agree with those obtained by Duncan (in press) for concurrent discriminations of location and various object features. The number of relevant objects is the crucial factor in this kind of visual divided attention; whether discriminations concern the same or different dimensions has no great effect.

Companion studies involving various surface dimensions have revealed one exception: Identifying the color of one object shows interference only from another concurrent surface discrimination. The explanation for this discrepancy is unclear; it may be noted, however, that weak interference between discriminations of color and shape was the basis for Allport's (1971) original support of the analyzer hypothesis.

This exception apart, the present results lend no support to the idea that subsystems dealing with different visual dimensions may be functionally parallel and independent. It seems impossible for one subsystem to produce output for one object $X$, while another produces output independently for a different object $Y$. Given the extensive evidence, neurophysiological and otherwise, for the separation of subsystems, the present findings have an interesting implication. As in Treisman and Souther's (1985) model, selective attention to a particular object must be a state in which subsystems are coordinated, enabling the representation of the whole object, with all of its attributes, for control of behavior.

\section{REFERENCES}

AllPORT, D. A. (1971), Parallel encoding within and between elementary stimulus dimensions. Perception \& Psychophysics, 10, 104-108.

Botella, J., Garcia, M. L., \& Barriopedro, M. (1992). Intrusion patterns in rapid serial visual presentation tasks with two response dimensions. Perception \& Psychophysics, 52, 547-552.

Broadbent, D. E. (1958). Perception and communication. London: Pergamon.

Broadbent, D. E. (1971). Decision and stress. London: Academic Press.

Desimone, R., \& Ungerleider, L. G. (1989). Neural mechanisms of visual processing in monkeys. In F. Boller \& J. Grafman (Eds.), Handbook of neuropsychology (Vol. 2, pp. 267-299). Amsterdam: Elsevier.

DUNCAN, J. (1984). Selective attention and the organization of visual information. Journal of Experimental Psychology: General, 113, 501-517.

DUNCAN, J. (in press). Coordination of what and where systems in visual attention. Perception.

Egeth, H., \& PaChella, R. (1969). Multidimensional stimulus identification. Perception \& Psychophysics, 5, 341-346.

Eriksen, C. W., \& Colegate, R. L. (1971). Selective attention and serial processing in briefly presented visual displays. Perception \& Psychophysics, 10, 321-326.

Kahneman, D., \& HeNIK, A. (1977). Effects of visual grouping on immediate recall and selective attention. In $\mathbf{S}$. Dornic (Ed.), Attention and performance VI (pp. 307-332). Hillsdale, NJ: Erlbaum. 
Kubovy, M. (1981). Concurrent pitch segregation: The theory of indispensable attributes. In M. Kubovy \& J. Pomerantz (Eds.), Perceptual organization (pp. 55-98). Hillsdale, NJ: Erlbaum.

LAPPIN, J. S. (1967). Attention in the identification of stimuli in complex displays. Journal of Experimental Psychology, 75, 321-328.

Livingstone, M. S., \& Hubel, D. H. (1987). Psychophysical evidence for separate channels for the perception of form, color, movement, and depth. Journal of Neuroscience, 7, 3416-3468.

LoNG, J. (1977). Division of attention: Evidence for a between-trial component of impaired performance. Quarterly Journal of Experimental Psychology, 29, 107-116.

Neisser, U. (1967). Cognitive psychology. New York: AppletonCentury-Crofts.

Nissen, M. J. (1985). Accessing features and objects: Is location special? In M. I. Posner \& O. S. M. Marin (Eds.), Attention and Performance $X I$ (pp. 205-219). Hillsdale, NJ: Erlbaum.

Rock, I., \& GuTMan, D. (1981). The effect of inattention on form perception. Journal of Experimental Psychology: Human Perception \& Performance, 7, 275-285.

Schwartz, E. L., Desimone, R., Albright, T. D., \& Gross, C. G. (1983). Shape recognition and inferior temporal neurons. Proceedings of the National Academy of Sciences U.S.A., 80, 5776-5778.

Shulman, G. L., \& WILson, J. (1987). Spatial frequency and selective attention to local and global information. Perception, 16, 89-101.

Treisman, A. M. (1969). Strategies and models of selective attention. Psychological Review, 76, 282-299.

Treisman, A., Kahneman, D., \& Burkell, J. (1983). Perceptual objects and the cost of filtering. Perception \& Psychophysics, 33, $527-532$.

Treisman, A., \& Souther, J. (1985). Search asymmetry: A diagnostic for preattentive processing of separable features. Journal of Experimental Psychology: General, 114, 285-310.

Ullman, S. (1984). Visual routines. Cognition, 18, 97-159.
Ungerleider, L. G., \& MishKin, M. (1982). Two cortical visual systems. In D. J. Ingle, M. A. Goodale, \& R. J. W. Mansfield (Eds.), Analysis of visual behaviour (pp. 549-586). Cambridge, MA: MIT Press.

VON WRIGHT, J. M. (1968). Selection in visual immediate memory. Quarterly Journal of Experimental Psychology, 20, 62-68.

WATT, R. (1988). Visual processing: Computational, psychophysical and cognitive research. Hillsdale, $\mathrm{NJ}$ : Erlbaum.

WING, A., \& AlLPORT, D. A. (1972). Multidimensional encoding of visual form. Perception \& Psychophysics, 12, 474-476.

\section{NOTES}

1. The rule of zero interference between discriminations concerning the same object does not hold when, for each discrimination, there are multiple response categories, approaching the limits of absolute judg ment (Egeth \& Pachella, 1969). In these cases, a limiting factor may be preserving multiple stable response criteria and mapping the perceputal impression onto them (Duncan, 1984; Long, 1977). Another exception to the rule was reported recently by Botella, Garcia, and Barriopedro (1992), whose subjects reported the color and/or identity of a single uppercase target word presented among a rapid sequence of differently colored, lowercase nontargets.

2. No attempt is made here to distinguish the number of relevant objects from the number of relevant locations; for evidence on this point, see Treisman et al. (1983) and Duncan (1984).

3. Using equivalent methods, Duncan (1992) found equal accuracies in single- and dual-discrimination conditions, providing that both discriminations concerned the same object. Thus, differences between conditions cannot be ascribed to response errors.

(Manuscript received December 9, 1992; revision accepted for publication March 4, 1993.) 